\title{
Analytical approximation of the exterior gravitational field of rotating neutron stars
}

\author{
C Teichmüller ${ }^{1}$, M B Fröb ${ }^{2}$ and F Maucher ${ }^{3}$ \\ 1 Theoretisch-Physikalisches Institut, University of Jena, Max-Wien-Platz 1, \\ 07743 Jena, Germany \\ 2 Departament de Física Fonamental, Institut de Ciències del Cosmos (ICC), \\ Universitat de Barcelona, C/Martí i Franquès 1, 08028 Barcelona, Spain \\ 3 Max Planck Institute for the Physics of Complex Systems, 01187 Dresden, \\ Germany \\ E-mail: christian.teichmueller@uni-jena.de
}

\begin{abstract}
It is known that Bäcklund transformations can be used to generate stationary axisymmetric solutions of Einstein's vacuum field equations with any number of constants. We will use this class of exact solutions to describe the exterior vacuum region of numerically calculated neutron stars. Therefore we study how an Ernst potential given on the rotation axis and containing an arbitrary number of constants can be used to determine the metric everywhere. Then we review two methods to determine those constants from a numerically calculated solution. Finally, we compare the metric and physical properties of our analytic solution with the numerical data and find excellent agreement even for a small number of parameters.

PACS numbers: 04.20-q, 04.25.D-, 04.40.Dg, 95.30.Sf, 97.60.Jd
\end{abstract}

\section{Introduction}

Solving Einstein's field equations in the vacuum with the assumption of axisymmetry and stationarity amounts to little more than solving Ernst's equation, which is a nonlinear partial differential equation. Ernst's formulation of the stationary axisymmetric problem (Ernst (1968a b) , Kramer and Neugebauer (1968)) allowed a reformulation of the stationary axisymmetric Einstein equations in terms of a "linear system" (see Maison (1978), Belinski and Zakharov (1978), Harrison (1978), Neugebauer (1979)), such that the integrability condition of the latter equals Ernst's equation.

During the past two decades, several papers have been published focussing on constructing analytical Ernst potentials and applying them to various settings (e.g. Manko and Sibgatullin (1993), Stute and Camenzind (2002), Berti and Stergioulas (2004) and recently Pappas (2009)). These include solutions to generalizations of Ernst's equation, which permit an electromagnetic field and use given axis data in the form of rational functions with an arbitrary number of constants on the axis of symmetry based on Sibgatullin's method (Sibgatullin (1991)).

Here, we will present an alternative approach using Bäcklund transformations acting w.l.o.g. on the Minkowski seed solution (see Neugebauer (1979), in particular Neugebauer $(1980 a)$ ) to find concise formulae for the determination of the involved parameters. This approach will result in an extremely simple, but in our opinion at 
the same time elegant recipe, that does not require any sophisticated mathematical background.

This method will then be used to calculate an analytical solution of the field equations in vacuum, which approximates the exterior metric for a given model of a rotating neutron star very accurately.

Two different approaches to compute the axis potential are considered. The resulting analytical solutions are then compared with rigorous numerical results using the AKM programme (Ansorg et al. (2003)) in the whole vacuum region. We find excellent agreement and convergence even for a small number of parameters.

This paper is organized as follows. In the first part, we review the properties of stationary and axisymmetric spacetimes and the relation between the metric and Ernst's potential. After that, we introduce the linear problem and show how to solve it using Bäcklund transformations with an arbitrary number of constants, referred to as Bäcklund parameters. Then we provide concise formulae determining those parameters from the axis potential. In the next part we illustrate two methods to compute the axis potential, namely by using Geroch-Hansen multipole moments and alternatively by fitting a quotient of polynomials to given axis values. After that we compare our analytical results with full numerical ones.

\section{Construction of the metric by Bäcklund transformations acting on a seed solution}

\subsection{The Ernst equation}

The exterior vacuum gravitational field of a stationary axisymmetric uniformly rotating matter configuration can be written in Weyl-Lewis-Papapetrou coordinates

$$
\mathrm{d} s^{2}=\mathrm{e}^{-2 U}\left[\mathrm{e}^{2 k}\left(\mathrm{~d} \varrho^{2}+\mathrm{d} \zeta^{2}\right)+\varrho^{2} \mathrm{~d} \varphi^{2}\right]-\mathrm{e}^{2 U}(\mathrm{~d} t+a \mathrm{~d} \varphi)^{2},
$$

where $U$ denotes the "Newtonian" gravitational potential, $a$ the "gravitomagnetic" potential and $k$ the superpotential, cf. e.g. Neugebauer and Meinel (2003). These three potentials are functions of $\varrho$ and $\zeta$ alone. The metric (1) admits an Abelian group of motions with the Killing vectors $\xi^{i}=\delta_{t}^{i}, \xi^{i} \xi_{i} \rightarrow-1$ asymptotically as $\varrho^{2}+\zeta^{2} \rightarrow \infty$ and $\eta^{i}=\delta_{\varphi}^{i}, \eta^{i} \eta_{i}>0$ for $\varrho>0$. $\delta_{j}^{i}$ is the Kronecker delta, so that $\xi^{i}$ has only a $t$-component, and $\eta^{i}$ points in the azimuthal $\varphi$-direction. Note that $U$ and $a$ can be related to scalar products of the Killing vectors by the coordinate independent expressions $\mathrm{e}^{2 U}=-\xi^{i} \xi_{i}$ and $a=-\mathrm{e}^{-2 U} \eta_{i} \xi^{i}$.

In the vacuum, Einstein's equations for $U$ and $a$ are equal to the so called Ernst equation:

$$
\Re(f)\left(f_{, \varrho \varrho}+f_{, \zeta \zeta}+\frac{1}{\varrho} f_{, \varrho}\right)=f_{, \varrho}^{2}+f_{, \zeta}^{2} .
$$

The metric coefficients $a, k, U$ and Ernst's potential are related via

$$
f(\varrho, \zeta)=\mathrm{e}^{2 U}+\mathrm{i} b,
$$

where $b$ was introduced as

$$
a_{, \varrho}=\varrho \mathrm{e}^{-4 U} b_{, \zeta}, \quad a_{, \zeta}=-\varrho \mathrm{e}^{-4 U} b_{, \varrho}
$$

and $k$ can be found by

$$
k_{, \varrho}=\varrho\left[U_{, \varrho}^{2}-U_{, \zeta}^{2}+\frac{1}{4} \mathrm{e}^{-4 U}\left(b_{, \varrho}^{2}-b_{, \zeta}^{2}\right)\right],
$$


Analytical approximation of the exterior gravitational field of rotating neutron stars 3

$$
k_{, \zeta}=2 \varrho\left[U_{, \varrho} U_{, \zeta}+\frac{1}{4} \mathrm{e}^{-4 U}\left(b_{, \varrho} b_{, \zeta}\right)\right] .
$$

It follows from (21), that $a_{, \varrho \zeta}=a_{, \zeta \varrho}$ and $k_{, \varrho \zeta}=k_{, \zeta \varrho}$ are fulfilled, so $a$ and $k$ can be obtained by line integration from $f$.

\subsection{The linear Problem}

It is well-known (see e.g. Neugebauer (1980b), Neugebauer and Kramer (1983)) that Ernst's equation is the integrability condition $\Phi_{, z \bar{z}}=\Phi_{, \bar{z} z}$ of the linear problem

$$
\begin{aligned}
& \Phi_{, z}=\left[\left(\begin{array}{cc}
B & 0 \\
0 & A
\end{array}\right)+\lambda\left(\begin{array}{cc}
0 & B \\
A & 0
\end{array}\right)\right] \Phi, \\
& \Phi_{, \bar{z}}=\left[\left(\begin{array}{cc}
\bar{A} & 0 \\
0 & \bar{B}
\end{array}\right)+\frac{1}{\lambda}\left(\begin{array}{cc}
0 & \bar{A} \\
\bar{B} & 0
\end{array}\right)\right] \Phi .
\end{aligned}
$$

Here, we introduced the complex coordinates $z:=\varrho+\mathrm{i} \zeta, \bar{z}:=\varrho-\mathrm{i} \zeta$ and the spectral parameter $\lambda:=\sqrt{(K-\mathrm{i} \bar{z}) /(K+\mathrm{i} z)}$, where $K$ is an arbitrary complex constant. $\Phi(\lambda, \bar{z}, z)$ is a $2 \times 2$ matrix, depending on the coordinates as well as on the spectral parameter, whereas $A$ and $B$ depend only on the coordinates $\varrho$ and $\zeta$. One may always multiply (5) from the right by a so-called gauge matrix which depends only on $K$. Equating all coefficients of $\lambda$ in the integrability condition leads to a set of 4 differential equations. One may introduce a potential $f$ in such a way that $B=\bar{f}_{, z} /(f+\bar{f})$ and $A=f_{, z} /(f+\bar{f})$. The remaining two equations are then the Ernst equation (2).

\subsection{Solving the linear system}

Solving a linear system, which depends on an additional spectral parameter, corresponds to solving a Riemann-Hilbert problem. The idea is then to discuss $\Phi$ as a holomorphic function of $\lambda$ for fixed $z$ and $\bar{z}$ and to calculate $A$ and $B$ afterwards. With the special ansatz that will be discussed in the following, no integrals have to be evaluated. Instead, solving the Riemann-Hilbert problem can be reduced to solving a linear system of equations. This special ansatz is to formulate a set of properties, such that if a matrix $\Phi$ has these properties, it automatically fulfils the linear problem.

A matrix $\Phi$ that has the properties (6-9) is a solution of the linear system (5) (Neugebauen (1996)).

$$
\Phi_{, z} \Phi^{-1}=Q+\lambda R, \quad \Phi_{, \bar{z}} \Phi^{-1}=S+T / \lambda,
$$

where $Q, R, S$ and $T$ are matrices that do not depend on $\lambda$.

$$
\begin{aligned}
& \Phi(\lambda)=\left(\begin{array}{cc}
\psi(\lambda) & \psi(-\lambda) \\
\chi(\lambda) & -\chi(-\lambda)
\end{array}\right) \\
& \overline{\psi\left(\bar{\lambda}^{-1}\right)}=\chi(\lambda) \\
& \psi(\lambda=-1, z, \bar{z})=\chi(\lambda=-1, z, \bar{z})=1
\end{aligned}
$$

For a detailed discussion see e.g. Maucher (2008). Neugebauer and Kramer (1983) used the ansatz

$$
\Phi=P \Phi_{0}=\mu \tilde{P} \Phi_{0}, \quad \text { with } \mu=\left(\frac{K+\mathrm{i} z}{K}\right)^{n}
$$


Analytical approximation of the exterior gravitational field of rotating neutron stars 4

to seek new solutions $\Phi$ from old ones $\Phi_{0}$, where

$$
\tilde{P}=\sum_{k=0}^{2 n} P_{k} \lambda^{k}
$$

is a finite matrix polynomial in $\lambda$. We restrict ourselves w.l.o.g. to the Minkowski seed solution

$$
\Phi_{0}=\left(\begin{array}{cc}
\psi_{0} & \psi_{0} \\
\chi_{0} & -\chi_{0}
\end{array}\right)=\left(\begin{array}{cc}
1 & 1 \\
1 & -1
\end{array}\right) .
$$

The fundamental lemma of algebra gives

$$
\operatorname{det} \tilde{P}(\lambda)=\operatorname{det} \sum_{i=0}^{2 n} P_{i} \lambda^{i}=\beta(z, \bar{z}) \prod_{k=1}^{4 n}\left(\lambda-\lambda_{k}\right),
$$

where the zeros $\lambda_{k}$ are functions of $z$ and $\bar{z}$ :

$$
\lambda_{k}=\sqrt{\frac{K_{k}-\mathrm{i} \bar{z}}{K_{k}+\mathrm{i} z}}
$$

with complex parameters $K_{k}$.

Since $\operatorname{det} \Phi\left(\lambda_{k}\right)=\mu^{2} \operatorname{det} \tilde{P}\left(\lambda_{k}\right) \operatorname{det} \Phi_{0}=0$, there is a non-trivial eigenvector for each $k$. Solving this system of algebraic equations using Cramer's rule, one finds the $n$-soliton solution for the particular Minkowski seed solution $\Phi_{0}$ :

$$
\begin{aligned}
& \chi(\lambda, z, \bar{z})=\left(\frac{K+\mathrm{i} z}{K}\right)^{n} \frac{\mathbb{A}(\lambda)}{\mathbb{A}(\lambda=-1)} \text { with } \\
& \mathbb{A}(\lambda)=\left|\begin{array}{cccccc}
1 & -\lambda & \lambda^{2} & -\lambda^{3} & \cdots & \lambda^{2 n} \\
1 & \alpha_{1} \lambda_{1} & \lambda_{1}^{2} & \alpha_{1} \lambda_{1}^{3} & \cdots & \lambda_{1}^{2 n} \\
1 & \alpha_{2} \lambda_{2} & \lambda_{2}^{2} & \alpha_{2} \lambda_{2}^{3} & \cdots & \lambda_{2}^{2 n} \\
1 & \alpha_{3} \lambda_{3} & \lambda_{3}^{2} & \alpha_{3} \lambda_{3}^{3} & \cdots & \lambda_{3}^{2 n} \\
\vdots & \vdots & \vdots & \vdots & \ddots & \vdots \\
1 & \alpha_{2 n} \lambda_{2 n} & \lambda_{2 n}^{2} & \alpha_{2 n} \lambda_{2 n}^{3} & \cdots & \lambda_{2 n}^{2 n}
\end{array}\right| .
\end{aligned}
$$

Here, the Bäcklund parameters

$$
\alpha_{k}=-\frac{\chi\left(-\lambda_{k}\right)+\chi\left(\lambda_{k}\right)}{\chi\left(-\lambda_{k}\right)-\chi\left(\lambda_{k}\right)}
$$

were introduced. Equation (8) restricts the complex parameters to $K_{i}=\bar{K}_{i}$ and $\alpha_{i} \bar{\alpha}_{i}=1$ or $K_{i}=\bar{K}_{k}$ and $\alpha_{i} \bar{\alpha}_{k}=1$. The Ernst potential can be found by setting $\lambda=1$ in (10):

$$
f(\varrho, \zeta)=\chi(\lambda=1, \varrho, \zeta) .
$$

The solution (12) of the Ernst equation (2) was derived by Neugebauer (1979) by an iterated application of Bäcklund transformations to the Minkowski space (Neugebauer $(1980 b))$. Explicit formulae for the metric functions can be found in Appendix Appendix A 


\subsection{Recovering the Ernst potential from its axis data}

Thus far, we discussed how to generate general solutions to Ernst's equation in the whole $(\rho, \zeta)$-plane. It is well-known, that the axis values of the Ernst's potential uniquely determine the Ernst potential in the whole plane (cf. Hauser and Ernst (1981)). The expression for $f$ found in the last section is a quotient of two polynomials in $\lambda_{i}$, and on the axis, this expression becomes a rational function in $\zeta$. In this subsection, we will explore the correspondence between the so far (apart from the reality condition (8) ) arbitrary Bäcklund parameters $K_{i}, \alpha_{i}$ and a given Ernst potential on the upper $\zeta$-axis $\mathcal{A}^{+}$, denoted as

$$
f(\zeta)=\frac{\zeta^{n}+\sum_{j=1}^{n} a_{j} \zeta^{j-1}}{\zeta^{n}+\sum_{j=1}^{n} b_{j} \zeta^{j-1}}=\frac{Z(\zeta)}{N(\zeta)}
$$

In the following, we will refer to Ernst potentials, that are rational functions on the axis as "rational potentials". The formulae that will be presented in this subsection were found by Neugebauer. They have also been discussed in Maucher (2008) and found recently application in Neugebauer and Hennig (2009).

From (5) together with the definition of $A$ and $B$ as functions of the Ernst potential, one finds that the matrix $\Phi$ factorizes into a matrix depending solely on $\zeta$ times a matrix $D(K)$ depending only on $K$. The matrix $D(K)$ can be evaluated explicitly at the branch point $\mathcal{W}:=(\lambda=0, K=\zeta, \varrho=0)$, where $\lambda$ becomes single valued. From (10) at $\lambda=1$ follows that the parameters $K_{i}$ are the zeros of the polynomia $\emptyset \frac{1}{2}[Z(\zeta) \bar{N}(\bar{\zeta})+\bar{Z}(\bar{\zeta}) N(\zeta)]$. Combining these two results, one finds the mentioned decomposition into $K$ and $\zeta$ dependent parts on the upper axis $\mathcal{A}^{+}$:

$$
\begin{aligned}
& \Phi(K, \zeta)=\left(\begin{array}{cc}
\bar{f}(\zeta) & 1 \\
f(\zeta) & -1
\end{array}\right) D(K) \text { and } \\
& D(K)=\frac{1}{2 K^{n}}\left(\begin{array}{cc}
N(K)+\bar{N}(\bar{K}) & N(K)-\bar{N}(\bar{K}) \\
Z(K)-\bar{Z}(\bar{K}) & Z(K)+\bar{Z}(\bar{K})
\end{array}\right) .
\end{aligned}
$$

This allows us to show the following:

Let $f(\zeta)$ be a rational potential of the form (13) with arbitrary parameters $a_{i}, b_{i}$. Let furthermore $K_{i} \neq K_{j}$ and $\alpha_{i} \neq \alpha_{j} \forall i \neq j$. For any set of parameters $\left\{a_{i}, b_{i}\right\}$ there is exactly one set of parameters $\left\{K_{i}, \alpha_{i}\right\}$, such that the Bäcklund transformation $\mathcal{B}$ acting on the Minkowski seed solution results in an Ernst potential $f$

$$
\mathcal{B}\left[f_{0}=1, K_{i}, \alpha_{i}\right]=f
$$

that becomes the given rational potential on the axis. The Bäcklund parameters can be explicitly calculated by evaluating the expressions

$$
\begin{aligned}
& \frac{1}{2}[Z(\zeta) \bar{N}(\bar{\zeta})+\bar{Z}(\bar{\zeta}) N(\zeta)]=\prod_{i=1}^{2 n}\left(\zeta-K_{i}\right), \\
& \frac{\bar{N}\left(\bar{K}_{i}\right)}{N\left(K_{i}\right)}=-\frac{\bar{Z}\left(\bar{K}_{i}\right)}{Z\left(K_{i}\right)}=\alpha_{i} .
\end{aligned}
$$

Proof:

Evaluation of

$$
\Phi\left(K_{i}\right)\left(\Phi_{0}\right)^{-1}\left(\begin{array}{c}
-\alpha_{i} \\
1
\end{array}\right)=0
$$

$\ddagger$ One has to consider the complex continuation of the real functions $Z(\zeta)$ and $N(\zeta)$. 
leads to (15). This overdetermined system of equations for the $\alpha_{i}$ has solutions, iff $Z\left(K_{i}\right) \bar{N}\left(\bar{K}_{i}\right)+\bar{Z}\left(\bar{K}_{i}\right) N\left(K_{i}\right)=0$. Another way of proving this fact is by simply evaluating the expressions (15) and (14) using the representation of $f$ in form of determinants (e.g. (10)) at $\lambda=1$, and verifying that they are fulfilled.

q.e.d.

The general algorithm to determine $f(\varrho, \zeta)$ from $f(\varrho=0, \zeta)$ works as follows: First of all, one has to calculate the $K_{i}$ by evaluating (14). The fundamental lemma of algebra guarantees existence and uniqueness. Then one can easily find the $\alpha_{i}$ by evaluating (15). The equations (14) and (15) are universal in the sense that one does not need to know beforehand whether the $K_{i}$ or the $\alpha_{i}$ are complex or real, nor does the order of the polynomial matter. Equation (15) allows the calculation of $\alpha_{i}$ even if $K_{i}$ is a zero of $N$ or of $Z$. The result can be easily generalized by using Bernoulli's rule for coinciding zeros $\left(K_{i}=K_{j}\right.$ for $\left.i \neq j\right)$. The $\alpha_{i}$ have to be treated as usual functions of $K_{i}$.

\section{Different approaches of constructing rational Ernst potentials on the rotation axis}

\subsection{Reflectional symmetric Ernst potentials}

In Newtonian theory one can show that rotating fluid bodies in equilibrium always have reflectional symmetry (cf. Lichtenstein (1933)). The same symmetry is to be expected in Einsteinian theory (cf. Lindblom (1992)) and we assume it to exist for our purpose. Therefore we study the influence of reflectional symmetry before constructing rational potentials. As shown in Meinel and Neugebauer (1995) and Kordas (1995), this kind of symmetry of the metric is uniquely characterized by a simple relation for the Ernst potential $f$ on the upper part $\mathcal{A}^{+}$of the $\zeta$-axis:

$$
f(\zeta) \overline{f(-\zeta)}=1 \text {. }
$$

Condition (16) is fulfilled for all $\zeta$, iff $N(\zeta)=\overline{Z(-\zeta)}$. As shown in the proof of Theorem 2 of Kordas (1995), this is equivalent to the following conditions for the coefficients:

$$
b_{n}=-\bar{a}_{n}, \quad b_{n-1}=\bar{a}_{n-1} \quad \ldots \quad b_{1}=\left\{\begin{array}{cc}
\bar{a}_{1}, & n \text { even } \\
-\bar{a}_{1}, & n \text { odd }
\end{array} .\right.
$$

So we have reduced the $2 n$ complex parameters to $2 n$ real ones.

\subsection{Construction from multipole moments}

As a first method of generating a rational Ernst potential $f(\zeta)$ on the rotation axis, we refer to Manko and Ruiz (1998). They start from the expansion of the function $X(\zeta)$ at $\zeta=\infty$ :

$$
X(\zeta) \equiv \frac{1-f(\zeta)}{1+f(\zeta)}=\sum_{j=0}^{\infty} m_{j} \zeta^{-j-1},
$$

where the coefficients $m_{j}$ determine the Geroch-Hansen multipole moments $P_{j}$ (the explicit relations between $m_{j}$ and $P_{j}$ are given in Fodor et al. (1989)). After plugging 
in the ansatz (13) and evaluating the algebraic set of equations, which results from comparing the coefficients of the powers of $\zeta$ up to $n=2 N$ in (18), one ends up with

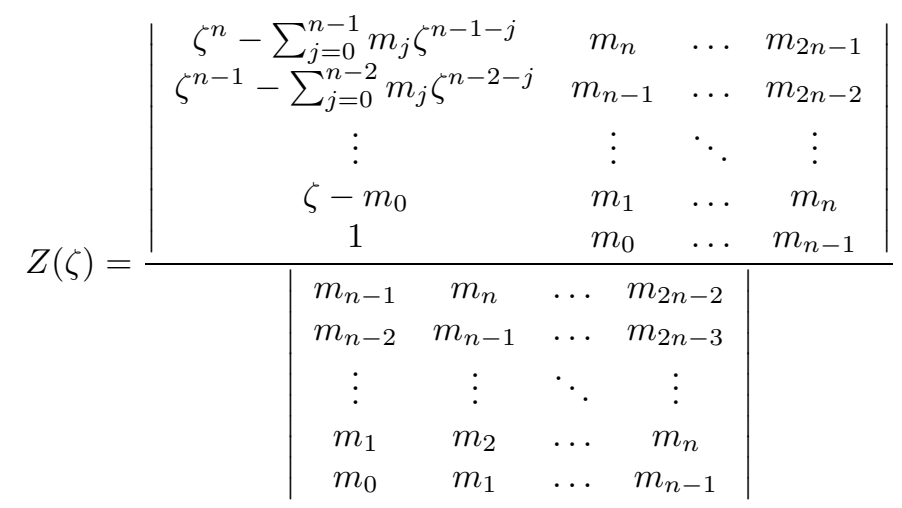

and an analogue formula for $N(\zeta)$ by changing the sign "-" to "+" in the first column of the numerator. From this formula one can see that condition (17) is automatically fulfilled when $m_{j}$ is real for even $j$ and purely imaginary otherwise, which is another well-known formulation of reflection symmetry (see Kordas (1995)).

Since only far-field information is employed by use of the multipole moments, it is guaranteed that the $f(\zeta)$ constructed in this way will coincide well with the solution from which the multipole moments were taken in a neighbourhood of infinity.

\subsection{Fitting a given (non-rational) Ernst potential}

Another method is constructing a rational potential $f(\zeta)$ from a given function $g(\zeta)$ on the rotation axis, which is especially useful in a numerical context. There, points $\zeta_{i}$ on the axis are selected where $f(\zeta)$ shall exactly coincide with $g(\zeta)$. If the points $\zeta_{i}$ are suitably chosen, we find a good approximation $f$ of $g$ on the whole axis. The same ansatz (13) is employed.

A major advantage of this method is that $f(\zeta)$ and $g(\zeta)$ will also coincide near the star's surface, if one or some of the $\zeta_{i}$ 's are situated there.

Rational potentials found this way do not automatically have the expected behaviour at large distances (all multipole moments and especially mass $M=m_{0}=P_{0}$ and angular momentum $J=-\mathrm{i} m_{1}=-\mathrm{i} P_{1}$ of the analytical solution may vary from the given numerical ones). To guarantee the right asymptotic behaviour, we treated the coefficients in (13) as functions of $m_{n}$ at fixed $M$ and $J$. The $m_{n}$ were varied until $f(\zeta)$ and $g(\zeta)$ coincided at the gridpoints $\zeta_{i}$.

\section{Results}

After constructing a rational Ernst potential one has to calculate the Bäcklund parameters according to formulae (14) and (15). For polynomials in numerator and denominator of degree $n$ there are $2 n$ real parameters. The analytical approximate solution (often referred to as Bäcklund solution, e.g. as presented in formula (10)) is a $2 n$-fold Bäcklund transformation acting on the Minkowski seed solution.

In the following we present solutions with $n=1,2,3$ and 4 to show the increasing accuracy of the approximation when taking more and more parameters into account.

For our numerical calculations we used the AKM programme (Ansorg et al. (2003)), where different coordinates had been introduced. We have chosen to compare 
our results in Weyl-Lewis-Papaetrou coordinates and have taken the necessary transformations into account.

\subsection{Comparison of metric functions}

As a first result we have calculated the metric functions and the relative difference between analytical and numerical values (see figure 1). For the numerical calculations one has to choose an equation of state. In this section we decided to model our neutron star to be composed of matter of constant energy density. The star we have chosen to treat as an example has a ratio of polar to equatorial radius of 0.7 (this ratio is always given in the coordinates used in Ansorg et al. (2003)) and a normalized central energy density of 1 in order to compare the results with Nozawa et al. (1998) and Ansorg et al. (2003).

$$
\begin{array}{lllllll}
\hline-\cdots n=1 & \cdots & n=2 & \cdots \cdots \cdot n=3 & \cdots \cdots \cdots n=4 \quad-A K M
\end{array}
$$
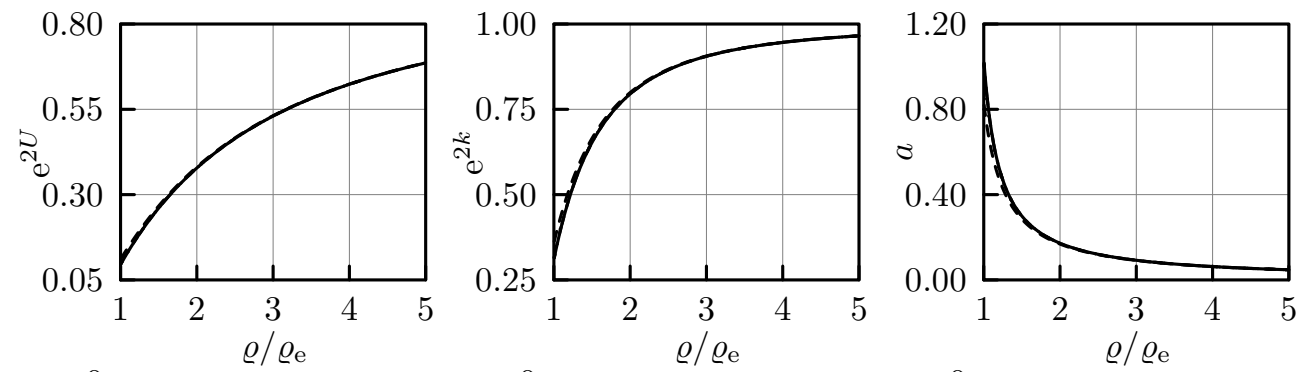

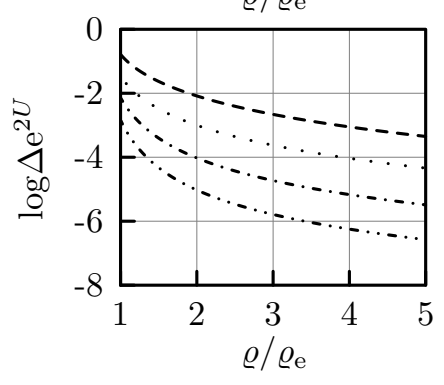

(a) Function $\mathrm{e}^{2 U}$ and relative difference for $\varrho=\varrho_{\mathrm{e}} \ldots 5 \varrho_{\mathrm{e}}$.

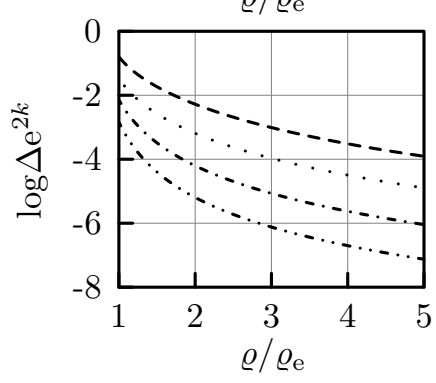

(b) Function $\mathrm{e}^{2 k}$ and relative difference for $\varrho=\varrho_{\mathrm{e}} \ldots 5 \varrho_{\mathrm{e}}$.

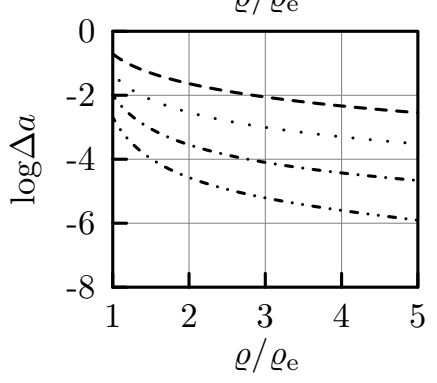

(c) Function $a$ and relative difference for $\varrho=\varrho_{\mathrm{e}} \ldots 5 \varrho_{\mathrm{e}}$.

Figure 1: Metric potentials as functions of coordinate radius $\varrho$ in the equatorial plane (homogeneous equation of state and fitted rational potential on the axis with fixed $M$ and $J)$.

These approximate solutions were constructed by fitting the given numerical Ernst potential on the axis, holding $M$ and $J$ fixed. For $n=1$ (i.e. a twofold Bäcklund transformation) this already determines the two available real parameters so that no additional gridpoint can be choosen. This is equivalent to approximating the neutron star's exterior metric by a Kerr solution with the same mass and angular momentum. We expect therefore the approximation to coincide well with the numerically calculated potentials only near infinity, as we already explained in the end of section 3.2. and to differ most near the star's surface.

In a $2 n$-fold Bäcklund transformation with $n>1$ one has to fix more parameters. 
In fitting the Ernst potential on the axis, our algorithm chooses a new gridpoint where - in the previously calculated $(2 n-1)$-fold transformation - the approximation and the numerical potential differ most. For a fourfold Bäcklund transformation, this is the star's surface, while for higher transformations the additional gridpoints are usually well spaced on the axis, which shows the efficiency of our algorithm.

As one may observe e.g. in figure 1, one gains about one order of magnitude in accuracy by going from a $n$-fold to a $(n+1)$-fold Bäcklund transformation, i.e. with two parameters more.

\subsection{Comparison of physical properties}

As we approximate the exterior vacuum region of a numerically calculated spacetime it is necessary to define what physical properties mean in our context. Of course one can simply read off mass $M$ and angular momentum $J$ from the far-field behaviour of the metric, as well as higher multipoles. This is sufficient if one only wants to analyse far-field behaviour.

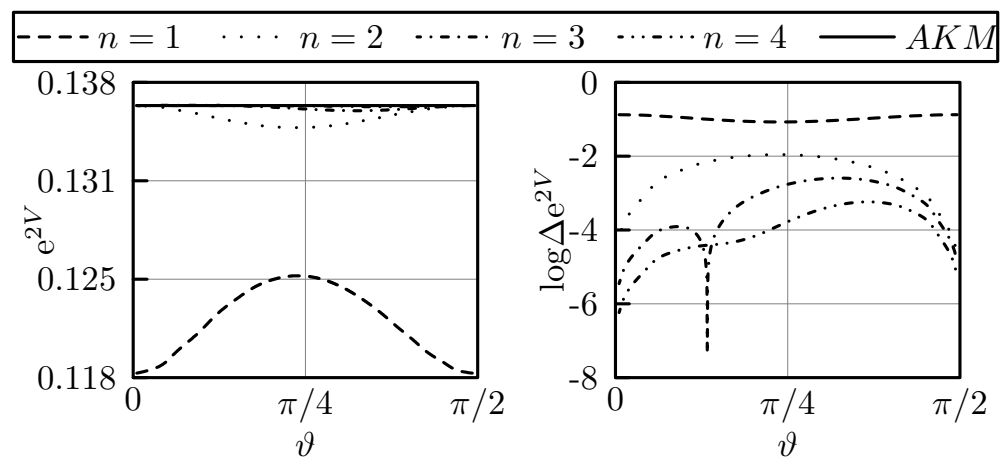

Figure 2: Potential $\mathrm{e}^{2 V}$ and relative difference as a function of $\vartheta:=\arctan (\varrho / \zeta) . \quad \mathrm{e}^{2 V}$ coincides at pole and equator (for $n>1$ ), because we obtained the analytical vacuum metric by fitting the numerical Ernst potential at the rotation axis (and the first gridpoint is the pole $\vartheta=0)$.

A more intriguing question is how to determine the star's surface in the approximated solution. Using the co-rotating potential $V \equiv U^{\prime}$ defined by (see e.g. Meinel et al. (2008))

$$
\mathrm{e}^{2 U^{\prime}}:=\mathrm{e}^{2 U}\left[(1+\Omega a)^{2}-\Omega^{2} \varrho^{2} \mathrm{e}^{-4 U}\right]
$$

the star's boundary is given by $V=V_{0}=$ const. However, given only the exterior metric (and not the rotation speed $\Omega$ of the star), it is not possible to construct the corotating potentials directly. Our approach to deal with this problem is the following: Since on the rotation axis $\varrho=0$ the co-rotating potentials coincide with the nonrotating ones, we read off the approximation of $V_{0}$ from the approximated potential $U$ at the north pole $V_{0}=U^{\prime}\left(\varrho=0, \zeta=\zeta_{\mathrm{p}}\right)=U\left(\varrho=0, \zeta=\zeta_{\mathrm{p}}\right)$ and use the constancy of $V_{0}$ to calculate the rotation speed $\Omega$ at the equator

$$
\mathrm{e}^{2 V_{0}}=\mathrm{e}^{2 U^{\prime}}\left(\varrho=\varrho_{\mathrm{e}}, \zeta=0\right) .
$$

The values $\varrho_{\mathrm{e}}$ and $\zeta_{\mathrm{p}}$ are supplied by the numerical solution. Once $\Omega$ is known we can evaluate $V \equiv U^{\prime}$ in the whole vacuum region and $V=V_{0}$ is valid at the pole and 
equator by construction. Of course, the so constructed $V$ will not be constant over the original (numerical) star's surface, and also $V_{0}$ will differ from the value in the numerically calculated solution if $U$ does not coincide at the pole. This can be seen in the change of behaviour from $n=1$ to $n=2$, since for $n>1$ a fitting gridpoint is located at the star's pole.

To illustrate this behaviour we plotted the potential $\mathrm{e}^{2 V}$ along the star's surface and the relative difference between the $2 n$-fold Bäcklund solution and the AKM solution in figure 2

To remedy also this non-constantness, we simply define the star's surface in our approximation as the region where $\mathrm{e}^{2 U^{\prime}}=\mathrm{e}^{2 V_{0}}$. One can see in figure 3 that this area is almost identical with the star's surface for solutions generated by $2 n$-fold Bäcklund transformations with $n>1$. This way of interpreting the analytical solution has the advantage that one only needs two surface points (the pole $\zeta_{\mathrm{p}}$ and the equator $\varrho_{\mathrm{e}}$ ) and does not need to worry about the whole surface function $\varrho_{s}(\zeta)$.

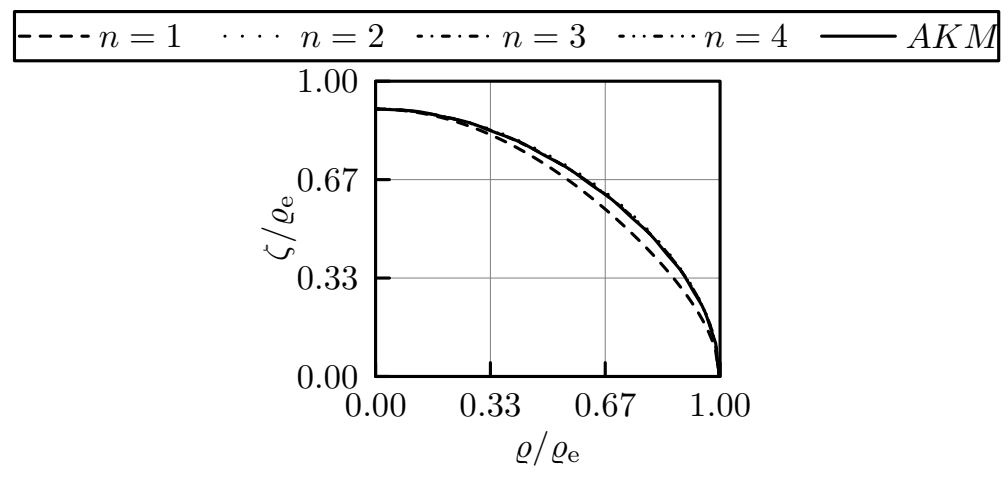

Figure 3: Area $\mathrm{e}^{2 U^{\prime}}=\mathrm{e}^{2 U}\left[(1+\Omega a)^{2}-\Omega^{2} \varrho^{2} \mathrm{e}^{-4 U}\right] \equiv \mathrm{e}^{2 V_{0}}$ in comparison with the star's surface.

Finally we calculated the following physical quantities in table 1 (formulae can be found in Nozawa et al. (1998) or Ansorg et al. (2003)):

$$
\begin{array}{ll}
R_{\text {circ }} & \text { equatorial circumferential radius } \\
Z_{\mathrm{p}} & \text { polar redshift } \\
Z_{\mathrm{eq}}^{\mathrm{f}} & \text { equatorial redshift in the forward direction } \\
Z_{\text {eq }}^{\mathrm{b}} & \text { equatorial redshift in the backward direction. }
\end{array}
$$

Analogous results for different equations of state can be found in Appendix Appendix B.

\subsection{Critical points and singularities}

A closer look at the spectral parameter $\lambda=\sqrt{(K-\mathrm{i} \bar{z}) /(K+\mathrm{i} z)}$, where the analogously defined $\lambda_{i}$ are part of the formulae for the metric functions, indicates some critical points, because the square root has a branch cut which we took along the negative real axis. One easily sees that the points

$$
z_{k 1}:=\mathrm{i} \bar{K}_{k}=\Im K_{k}+\mathrm{i} \Re K_{k},
$$

where the radicand of $\lambda_{k}$ vanishes and

$$
z_{k 2}:=\mathrm{i} K_{k}=-\Im K_{k}+\mathrm{i} \Re K_{k},
$$


Table 1: Detailed comparison of physical quantities (homogeneous equation of state $\left(\mu=\mu_{0}\right)$ and fitted rational potential on the axis with fixed $M$ and $\left.J\right)$. Here, $\tilde{p}_{\mathrm{c}}=p_{\mathrm{c}} / \mu_{0}, \tilde{\Omega}=$ $\Omega / \mu_{0}^{1 / 2}, \tilde{M}=M \mu_{0}^{1 / 2}, \tilde{R}_{\text {circ }}=R_{\text {circ }} \mu_{0}^{1 / 2}$ and $\tilde{J}=J \mu_{0}$ are normalized values of the physical quantities. The column labelled AKM shows numerically calculated values, whereas BT $[n]$ refers to values of a $2 n$-fold Bäcklund solution. $\Delta \mathrm{BT}[n]$ labels the relative difference between $\mathrm{BT}[n]$ and AKM.

\begin{tabular}{cccccccccc}
\hline Model & $\mathrm{AKM}$ & $\mathrm{BT}[1]$ & $\mathrm{BT}[2]$ & $\mathrm{BT}[3]$ & $\mathrm{BT}[4]$ & $\Delta \mathrm{BT}[1]$ & $\Delta \mathrm{BT}[2]$ & $\Delta \mathrm{BT}[3]$ & $\Delta \mathrm{BT}[4]$ \\
\hline$\tilde{p}_{\mathrm{c}}$ & 1.00 & & & & & & & & \\
$r_{\mathrm{p}} / r_{\mathrm{e}}$ & 0.700 & & & & & & & & \\
$\tilde{\Omega}$ & 1.41 & 1.58 & 1.42 & 1.41 & 1.41 & $1.2 \mathrm{e}-01$ & $7.4 \mathrm{e}-03$ & $1.4 \mathrm{e}-03$ & $2.9 \mathrm{e}-04$ \\
$\tilde{M}$ & 0.136 & 0.136 & 0.136 & 0.136 & 0.136 & & & & \\
$\tilde{J}$ & 0.0141 & 0.0141 & 0.0141 & 0.0141 & 0.0141 & & & & \\
$\tilde{R}_{\text {circ }}$ & 0.345 & 0.337 & 0.344 & 0.345 & 0.345 & $2.4 \mathrm{e}-02$ & $4.6 \mathrm{e}-03$ & $9.3 \mathrm{e}-04$ & $1.9 \mathrm{e}-04$ \\
$Z_{\mathrm{p}}$ & 1.71 & 1.91 & 1.71 & 1.71 & 1.71 & $1.2 \mathrm{e}-01$ & & & \\
$Z_{\text {eq }}^{\mathrm{f}}$ & -0.163 & -0.295 & -0.170 & -0.164 & -0.163 & $8.1 \mathrm{e}-01$ & $4.3 \mathrm{e}-02$ & $8.5 \mathrm{e}-03$ & $1.8 \mathrm{e}-03$ \\
$Z_{\text {eq }}^{\mathrm{b}}$ & 11.4 & 11.7 & 11.1 & 11.3 & 11.3 & $2.6 \mathrm{e}-02$ & $2.8 \mathrm{e}-02$ & $5.7 \mathrm{e}-03$ & $1.1 \mathrm{e}-03$ \\
\hline
\end{tabular}

where the radicand of $\lambda_{k}$ goes to infinity can become problematic. For example in the case of a 4 -fold Bäcklund transformation with 4 complex $K_{i}\left(K_{1}, K_{2}=\right.$ $\bar{K}_{1}, K_{3}=-\bar{K}_{1}$ and $K_{4}=-K_{1}$ ) the metric function $\mathrm{e}^{2 U}$ has a jump along $g:\left\{\varrho \in\left[0, \Im K_{1}\right], \zeta=\Re K_{1}\right\}$.

As the formulae for the calculation of the metric functions contain determinants in the denominator, even singularities of the analytic solution may occur. Therefore we have numerically calculated the zeros of the denominator (see figure 4) and roughly speaking they usually appear near or between the $K_{i}$.
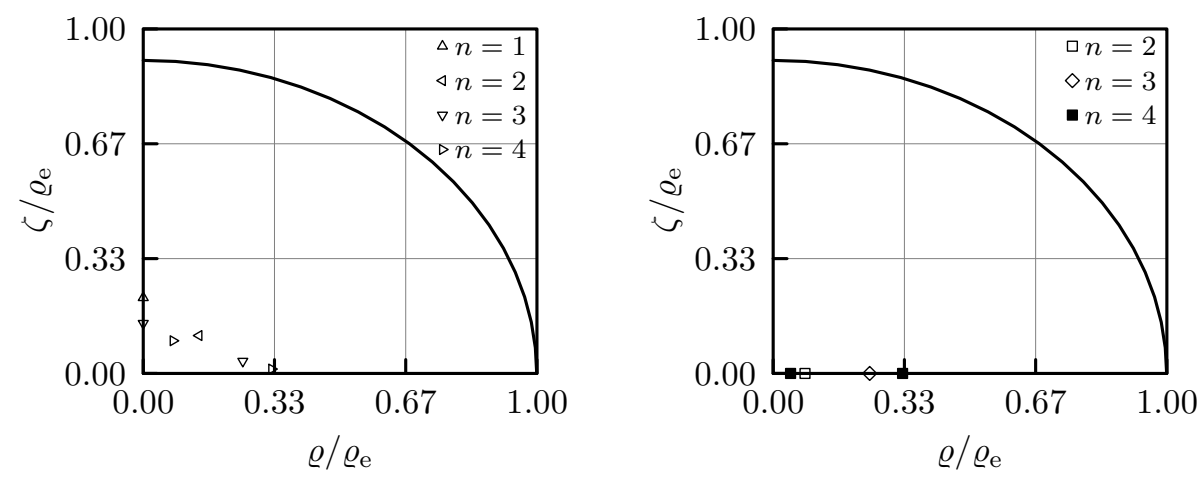

Figure 4: Star's surface (solid line), critical points (triangles) and singularities (boxes) of the Bäcklund solutions. The exterior solution is well behaved, because the difficult points are inside the star and the singularities are situated near the critical points.

All these problems are controllable for our purpose. As we search for an analytical vacuum solution for the exterior of a neutron star, this is well behaved as long as the critical points and singularities are located in the interior region of the star. To decide whether our solution is applicable or not, we simply added a plot of the star's surface with the critical points to the output of our approximation programme. 


\subsection{Numerical methods}

To calculate the approximation, we augmented the AKM programme Ansorg et al. (2003)) to incorporate the formulae given above. Two main tasks had to be done: Calculation of determinants (e.g. A.1), A.2) and (A.3) ) and root finding for polynomials (14).

To calculate the determinants, Gaussian forward elimination was used to convert the matrix to triangular form, whence the determinant is given by multiplication of the diagonal elements. For our purposes, this method was found to be numerically stable by using partial pivoting, i.e. transposing columns until the largest element in each row was on the diagonal.

Polynomial root finding was accomplished for $n=2$ and $n=3$ by direct solving the quadratic or cubic equation, while for $n>3$ we determined the roots as the eigenvalues of the companion matrix. However, Gaussian elimination was not sufficient in this case and we had to resort to a QR decomposition of the companion matrix (adapted from the GSL (cf. Galassi et al. (2009))).

At last, to find the singularities, we had to search for zeros of the denominator in (A.1). Since the determinant $\mathbb{A}^{+}$, viewed as function in the complex $(\rho, \zeta)$-plane, has branch cuts where it is not even continous, the obvious Newton's method fails spectacularly. The Nelder-Mead simplex algorithm (Nelder and Mead (1965)) does work for non-continuous functions and is well behaved for this problem. We start with a number of random trial points in the $(\rho, \zeta)$-plane, from which this algorithm searches local minima of the absolute value of the determinant. Afterwards we check if these minima really are zeros.

\subsection{Conclusion}

It is remarkable that the whole exterior region of a stationary and axisymmetric neutron star can be expressed with an accuracy greater than $99 \%$ everywhere using only 6 to 10 real parameters $\left(\varrho_{\mathrm{e}}\right.$ and $\zeta_{\mathrm{p}}$ to determine the star's surface and 4 to 8 multipole moments, $m_{n}$ or values of Ernst's potential on the rotation axis). As there are many ways to construct a rational potential on the axis one may choose whether near or far-field information should be considered. From this point of view, there is no "best approximation", but rather it can be adjusted for specific needs.

In each case one has to be aware of the singularities and as long as they are located in the interior of the star, there is no problem.

\section{Appendix A. Explicit formulae for the determination of metric functions}

An alternative formula (obtainable from (10) by performing the limit $\lambda \rightarrow 1$, some manipulation of the determinants and inserting the definition of $r_{i}$ ) for the calculation of Ernst's potential is

$$
f(\varrho, \zeta)=\frac{\mathbb{A}^{-}}{\mathbb{A}^{+}}, \quad \mathbb{A}^{\mp}=\left|\begin{array}{ccccc}
1 & \mp 1 & 0 & \ldots & 0 \\
K_{1}^{n} & K_{1}^{n-1} \alpha_{1} r_{1} & K_{1}^{n-1} & \ldots & 1 \\
K_{2}^{n} & K_{2}^{n-1} \alpha_{2} r_{2} & K_{2}^{n-1} & \ldots & 1 \\
\vdots & \vdots & \vdots & \ddots & \vdots \\
K_{2 n}^{n} & K_{2 n}^{n-1} \alpha_{2 n} r_{2 n} & K_{2 n}^{n-1} & \ldots & 1
\end{array}\right|,(\mathrm{A} .1)
$$


with $r_{i}=\lambda_{i}\left(K_{i}+\mathrm{i} z\right)$. Of course, $\mathrm{e}^{2 U}$ is the real part of $f$ and one could obtain $a$ from its imaginary part $b$ via (3). A straightforward calculation leads to the explicit formula

$$
\begin{aligned}
& \left(a-a_{0}\right) \mathrm{e}^{2 U}=2 n \varrho+2 \Re\left(\frac{\mathbb{B}}{\mathbb{A}^{+}}\right), \\
& \mathbb{B}=\left|\begin{array}{cccccc}
-n z & -\varrho-(n-1) z & -\mathrm{i} & -\mathrm{i} & \ldots & 0 \\
K_{1}^{n} & \alpha_{1} r_{1} K_{1}^{n-1} & K_{1}^{n-1} & \alpha_{1} r_{1} K_{1}^{n-2} & \ldots & 1 \\
K_{2}^{n} & \alpha_{2} r_{2} K_{2}^{n-1} & K_{2}^{n-1} & \alpha_{2} r_{2} K_{2}^{n-2} & \ldots & 1 \\
\vdots & \vdots & \vdots & \vdots & \ddots & \vdots \\
K_{2 n}^{n} & \alpha_{2 n} r_{2 n} K_{2 n}^{n-1} & K_{2 n}^{n-1} & \alpha_{2 n} r_{2 n} K_{2 n}^{n-2} & \ldots & 1
\end{array}\right|,
\end{aligned}
$$

where the integration is already done. The only thing left to be done is the determination of the constant $a_{0}$, which can be calculated by demanding that $a \rightarrow 0$ as $\varrho \rightarrow \infty$.

Furthermore the last metric function $\mathrm{e}^{2 k}$ could be calculated via line integration of (41). By reformulating the linear problem (5) and some manipulation of determinants (cf. Kramer et al. (1991), Kramer (1980)) one ends up with the following formula:

$$
\begin{aligned}
& \mathrm{e}^{2\left(k-k_{0}\right)}=\prod_{j=1}^{2 n} \frac{1}{r_{j}\left(K_{j}+\mathrm{i} z\right)} \mathbb{D}_{1} \mathbb{D}_{2}, \\
& \mathbb{D}_{1}=\left|\begin{array}{cccc}
\left(K_{1}+\mathrm{i} z\right)^{n} & \alpha_{1} r_{1} K_{1}^{n-1} & \ldots & \alpha_{1} r_{1} \\
\left(K_{2}+\mathrm{i} z\right)^{n} & \alpha_{2} r_{2} K_{2}^{n-1} & \ldots & \alpha_{2} r_{2} \\
\vdots & \vdots & \ddots & \vdots \\
\left(K_{2 n}+\mathrm{i} z\right)^{n} & \alpha_{2 n} r_{2 n} K_{2 n}^{n-1} & \ldots & \alpha_{2 n} r_{2 n}
\end{array}\right|
\end{aligned}
$$

and $\mathbb{D}_{2}$ is the same as $\mathbb{D}_{1}$ with $\alpha_{i}$ replaced by $1 / \alpha_{i}$. Finally the integration constant $k_{0}$ can be evaluated by demanding that $\mathrm{e}^{2 k} \rightarrow 1$ as $\varrho \rightarrow \infty$.

\section{Appendix B. Results for different equations of state and approximation methods}

In order to avoid a flood of data we do not present the results for three different equations of state (strange quark, constant energy density and polytropic) with three different approximation methods (via moments $m_{n}$, fitting a nonrational potential and fitting a nonrational potential with fixed $M$ and $J$ ). The following figures and tables provide one example of each method, but not necessarily the best one for the given equation of state.

Appendix B.1. Polytropic stars approximated by moments $m_{n}$

As an example with polytropic equation of state (polytropic exponent $\Gamma=2$, polytropic constant $K$ ) we have chosen $\tilde{\mu}_{\mathrm{c}}=1$ and $r_{\mathrm{p}} / r_{\mathrm{e}}=0.834$ in order to compare the values for the physical quantities with Nozawa et al. (1998) and Ansorg et al. (2003).

A first look at figure B1 shows, that all approximated functions coincide with errors less than 1\%. The eightfold Bäcklund solution is missing from those data. Since critical points and singularities appeared outside the star, we had to discard this 

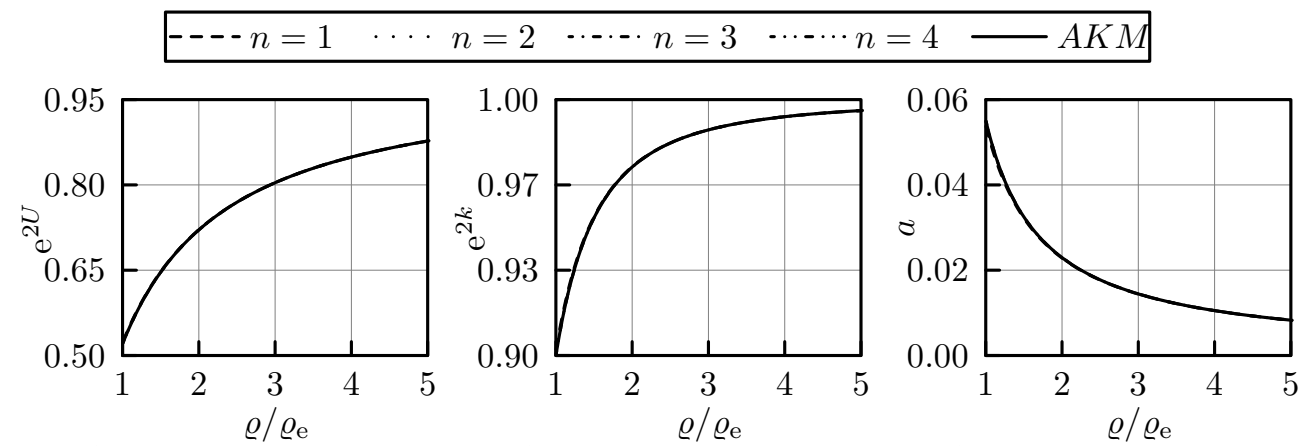

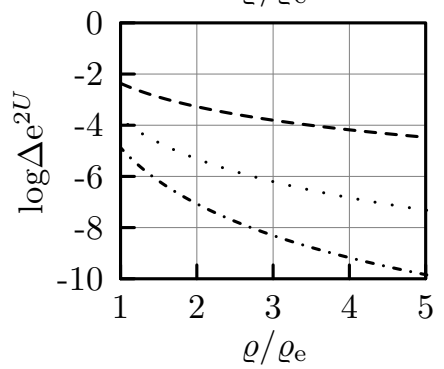

(a) Function $\mathrm{e}^{2 U}$ and relative difference for $\varrho=\varrho_{\mathrm{e}} \ldots 5 \varrho_{\mathrm{e}}$.

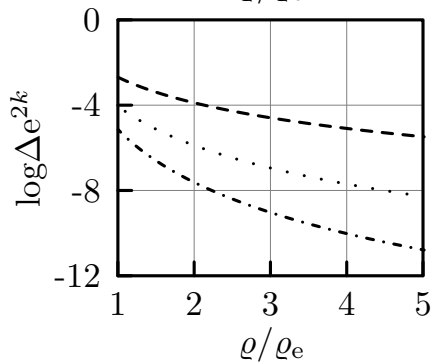

(b) Function $\mathrm{e}^{2 k}$ and relative difference for $\varrho=\varrho_{\mathrm{e}} \ldots 5 \varrho_{\mathrm{e}}$.

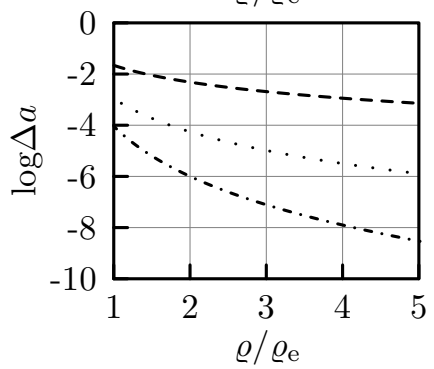

(c) Function $a$ and relative difference for $\varrho=\varrho_{\mathrm{e}} \ldots 5 \varrho_{\mathrm{e}}$.

Figure B1: Metric potentials as functions of coordinate radius $\varrho$ in the equatorial plane (polytropic equation of state and rational potential via moments $m_{n}$ on the axis).

solution. As these approximated solutions are generated only by far-field information we observed quite generally that critical points appear more often outside the star.

As this approximation method does not include any information about the star's surface, at first sight one could think that $\mathrm{e}^{2 V}$ is not approximated very well. The graphs (see figure B2 however show quite a good approximation even in this case (especially for $n>1$ ). Remembering the definition of $V_{0}$ and $\Omega$ (cf. (201) ) it is clear that the values of $\mathrm{e}^{2 V}$ for $\vartheta=0$ and $\vartheta=\pi / 2$ coincide.
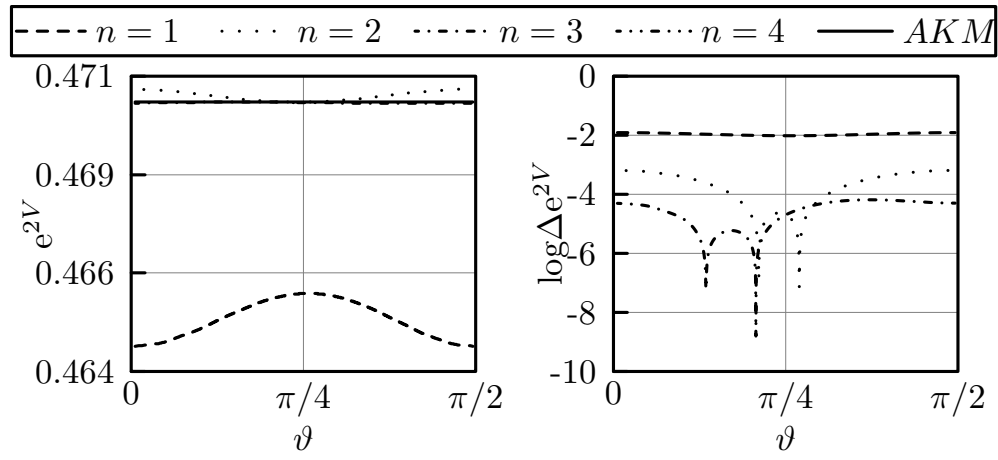

Figure B2: Potential $\mathrm{e}^{2 V}$ as a function of $\vartheta:=\arctan (\varrho / \zeta)$.

Figure B3 confirms that the real star's surface coincides well with the one determined in the approximation by $\mathrm{e}^{2 U^{\prime}} \equiv \mathrm{e}^{2 V_{0}}$. 
Analytical approximation of the exterior gravitational field of rotating neutron stars 15

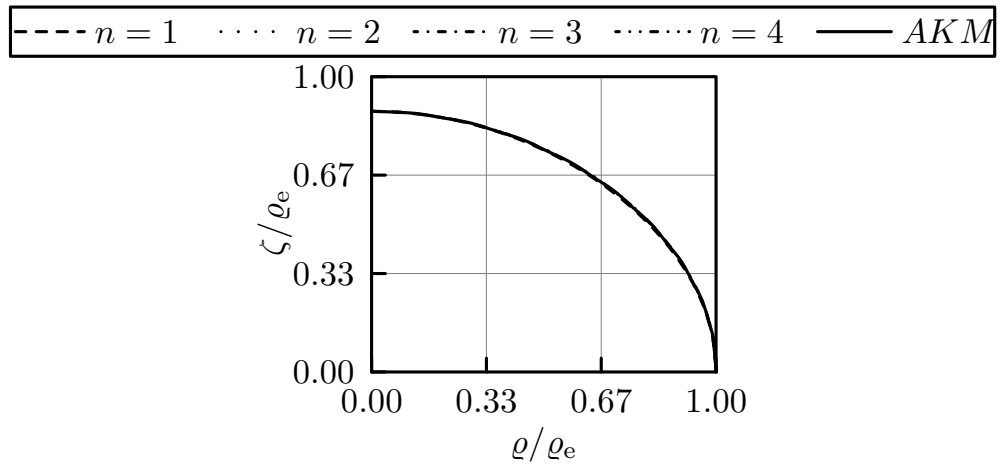

Figure B3: Area $\mathrm{e}^{2 U^{\prime}}=\mathrm{e}^{2 U}\left[(1+\Omega a)^{2}-\Omega^{2} \varrho^{2} \mathrm{e}^{-4 U}\right] \equiv \mathrm{e}^{2 V_{0}}$ in comparison with the star's surface.

Finally, we calculated some physical quantities in table B1

Table B1: Detailed comparison of physical quantities (polytropic equation of state $(\mu=p+\sqrt{p / K})$ and rational potential via $\left.m_{n}\right)$. Here, $\tilde{\mu}_{\mathrm{c}}=\mu_{\mathrm{c}} K, \tilde{\Omega}=\Omega K^{1 / 2}, \tilde{M}=$ $M / K^{1 / 2}, \tilde{R}_{\text {circ }}=R_{\text {circ }} / K^{1 / 2}$ and $\tilde{J}=J / K$ are normalized values of the physical quantities. The column labelled AKM shows numerically calculated values, whereas BT $[n]$ refers to values of a $2 n$-fold Bäcklund solution. $\Delta \mathrm{BT}[n]$ labels the relative difference between $\mathrm{BT}[n]$ and AKM.

\begin{tabular}{cccccccc}
\hline Model & AKM & BT[1] & BT[2] & BT[3] & $\Delta \mathrm{BT}[1]$ & $\Delta \mathrm{BT}[2]$ & $\Delta \mathrm{BT}[3]$ \\
\hline$\tilde{\mu}_{\mathrm{c}}$ & 1.00 & & & & & & \\
$r_{\mathrm{p}} / r_{\mathrm{e}}$ & 0.834 & & & & & & \\
$\tilde{\Omega}$ & 0.400 & 0.425 & 0.400 & 0.401 & $6.1 \mathrm{e}-02$ & $1.8 \mathrm{e}-03$ & $2.4 \mathrm{e}-04$ \\
$\tilde{M}$ & 0.161 & 0.161 & 0.161 & 0.161 & & & \\
$\tilde{J}$ & $9.49 \mathrm{e}-03$ & $9.49 \mathrm{e}-03$ & $9.49 \mathrm{e}-03$ & $9.49 \mathrm{e}-03$ & & & \\
$\tilde{R}_{\text {circ }}$ & 0.679 & 0.678 & 0.679 & 0.679 & $2.1 \mathrm{e}-03$ & $8.2 \mathrm{e}-05$ & $5.8 \mathrm{e}-06$ \\
$Z_{\mathrm{p}}$ & 0.458 & 0.467 & 0.458 & 0.458 & $2.0 \mathrm{e}-02$ & $1.0 \mathrm{e}-03$ & $8.0 \mathrm{e}-05$ \\
$Z_{\mathrm{eq}}^{\mathrm{f}}$ & -0.0601 & -0.0845 & -0.0594 & -0.0602 & $4.1 \mathrm{e}-01$ & $1.2 \mathrm{e}-02$ & $1.6 \mathrm{e}-03$ \\
$Z_{\text {eq }}^{\mathrm{b}}$ & 1.04 & 1.09 & 1.04 & 1.04 & $4.4 \mathrm{e}-02$ & $1.8 \mathrm{e}-03$ & $1.8 \mathrm{e}-04$ \\
\hline
\end{tabular}

Appendix B.2. Strange quark stars approximated by fitting Ernst's potential at the rotation axis

As an example with strange quark equation of state (linear relation between energy density and pressure, MIT-Bag constant $B$ ) we have chosen $\tilde{p}_{\mathrm{c}}=2$ and $r_{\mathrm{p}} / r_{\mathrm{e}}=0.5$ in order to compare the values for the physical quantities with Ansorg et al. (2003). As the star is quite relativistic, figure B4 shows that all approximated functions do not reach the accuracy of the other examples.

The next figure shows the area $\mathrm{e}^{2 U^{\prime}} \equiv \mathrm{e}^{2 V_{0}}$. The fact that the curve for the $n=1$ Bäcklund solution does not end at the equatorial radius, might be confusing at the first sight (especially when in figure B5 the error of $\mathrm{e}^{2 V}$ vanishes there). As the surface area $\mathrm{e}^{2 U^{\prime}}$ is not necessarily a monotonic function, there can be two intersection curves 
Analytical approximation of the exterior gravitational field of rotating neutron stars 16

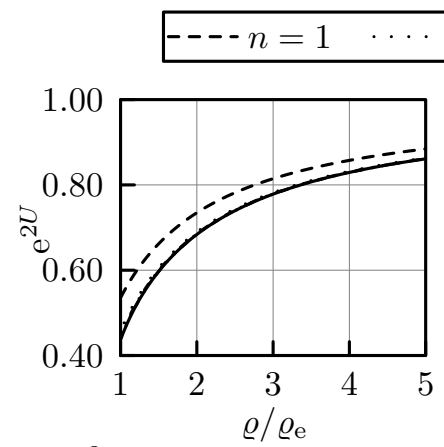

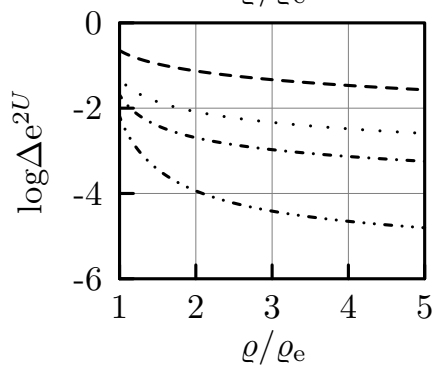

(a) Function $\mathrm{e}^{2 U}$ and relative difference for $\varrho=\varrho_{\mathrm{e}} \ldots 5 \varrho_{\mathrm{e}}$.
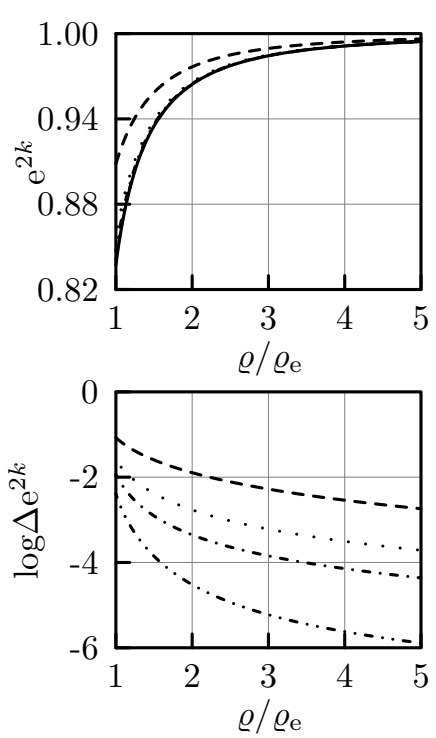

(b) Function $\mathrm{e}^{2 k}$ and relative difference for $\varrho=\varrho_{\mathrm{e}} \ldots 5 \varrho_{\mathrm{e}}$.
$A K M$
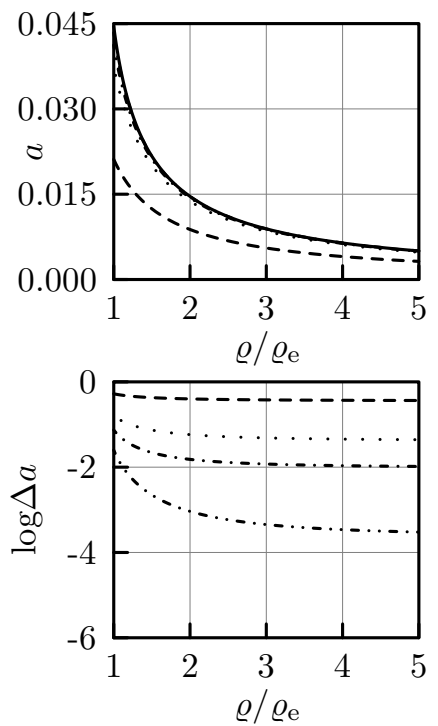

(c) Function $a$ and relative difference for $\varrho=\varrho_{\mathrm{e}} \ldots 5 \varrho_{\mathrm{e}}$.

Figure B4: Metric potentials as functions of coordinate radius $\varrho$ in the equatorial plane (strange quark equation of state and fitted rational potential on the axis).

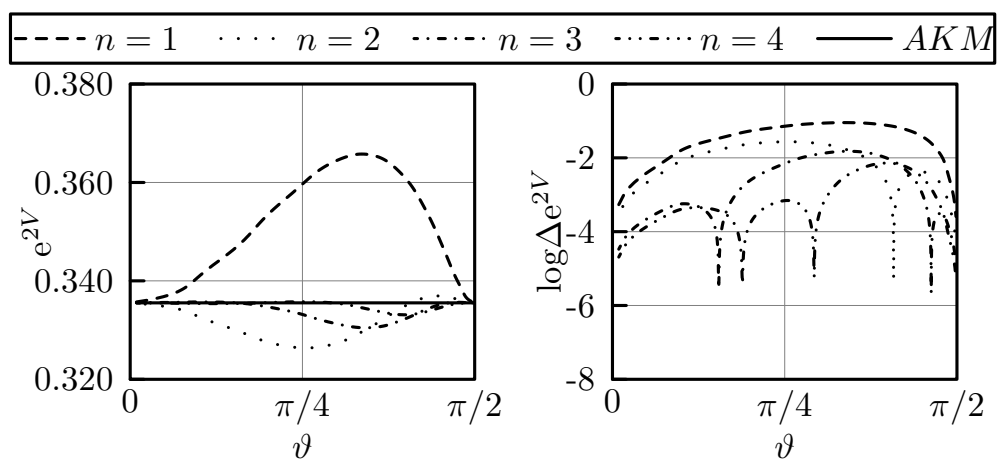

Figure B5: Potential $\mathrm{e}^{2 V}$ as a function of $\vartheta:=\arctan (\varrho / \zeta) \cdot \mathrm{e}^{2 V}$ coincides at pole and equator, because we obtained the analytical vacuum metric by fitting the numerical Ernst's potential at the rotation axis (and the first gridpoint is the pole $\vartheta=0$ ).

that need not be connected. In figure B6 this is the case, where one intersection curve ends at $\varrho \approx 0.67 \varrho_{\mathrm{e}}$ and the other starts at $\varrho=\varrho_{\mathrm{e}}$.

Finally, we calculated some physical quantities in table B2.

\section{Acknowledgments}

This work has been supported by the DFG under GRK1523. 
Analytical approximation of the exterior gravitational field of rotating neutron stars 17

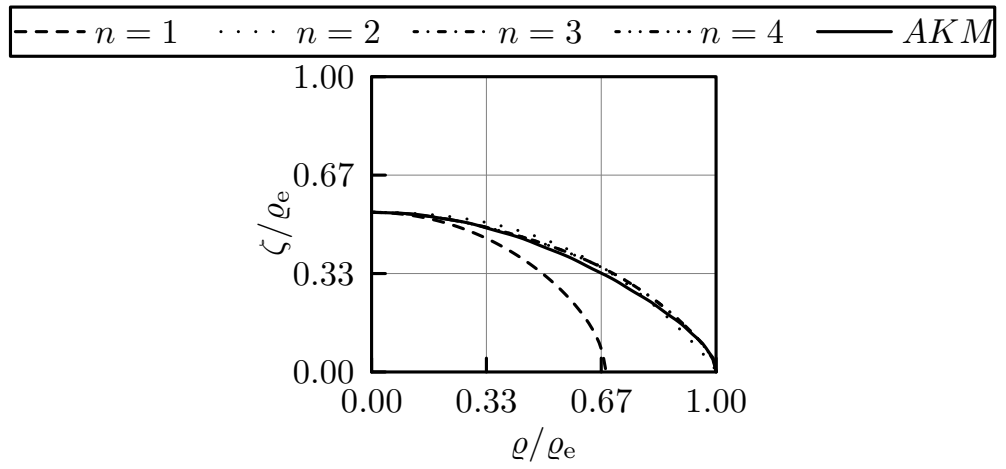

Figure B6: Area $\mathrm{e}^{2 U^{\prime}}=\mathrm{e}^{2 U}\left[(1+\Omega a)^{2}-\Omega^{2} \varrho^{2} \mathrm{e}^{-4 U}\right] \equiv \mathrm{e}^{2 V_{0}}$ in comparison with the star's surface.

Table B2: Detailed comparison of physical quantities (strange matter equation of state $(\mu=3 p+4 B)$ and fitted rational potential on the axis). Here, $\tilde{p}_{\mathrm{c}}=p_{\mathrm{c}} / B, \tilde{\Omega}=\Omega / B^{1 / 2}, \tilde{M}=$ $M B^{1 / 2}, \tilde{R}_{\text {circ }}=R_{\text {circ }} B^{1 / 2}$ and $\tilde{J}=J B$ are normalized values of the physical quantities. The column labelled AKM shows numerically calculated values, whereas BT $[n]$ refers to values of a $2 n$-fold Bäcklund solution. $\Delta \mathrm{BT}[n]$ labels the relative difference between BT $[n]$ and AKM.

\begin{tabular}{cccccccccc}
\hline Model & AKM & BT[1] & BT[2] & BT[3] & BT[4] & $\Delta \mathrm{BT}[1]$ & $\Delta \mathrm{BT}[2]$ & $\Delta \mathrm{BT}[3]$ & $\Delta \mathrm{BT}[4]$ \\
\hline$\tilde{p}_{\mathrm{c}}$ & 2.00 & & & & & & & & \\
$r_{\mathrm{p}} / r_{\mathrm{e}}$ & 0.500 & & & & & & & & \\
$\tilde{\Omega}$ & 3.43 & 4.20 & 3.57 & 3.48 & 3.45 & $2.2 \mathrm{e}-01$ & $4.0 \mathrm{e}-02$ & $1.5 \mathrm{e}-02$ & $4.3 \mathrm{e}-03$ \\
$\tilde{M}$ & 0.0355 & 0.0293 & 0.0349 & 0.0354 & 0.0355 & $1.8 \mathrm{e}-01$ & $1.6 \mathrm{e}-02$ & $3.6 \mathrm{e}-03$ & $8.3 \mathrm{e}-05$ \\
$\tilde{J}$ & $1.10 \mathrm{e}-03$ & $7.12 \mathrm{e}-04$ & $1.06 \mathrm{e}-03$ & $1.92 \mathrm{e}-03$ & $1.10 \mathrm{e}-03$ & $3.5 \mathrm{e}-01$ & $4.1 \mathrm{e}-02$ & $9.6 \mathrm{e}-03$ & $3.3 \mathrm{e}-04$ \\
$\tilde{R}_{\text {circ }}$ & 0.141 & 0.129 & 0.139 & 0.140 & 0.141 & $8.4 \mathrm{e}-02$ & $1.9 \mathrm{e}-02$ & $7.8 \mathrm{e}-03$ & $2.4 \mathrm{e}-03$ \\
$Z_{\mathrm{p}}$ & 0.726 & 0.726 & 0.726 & 0.726 & 0.726 & & & & \\
$Z_{\mathrm{eq}}^{\mathrm{f}}$ & -0.302 & -0.411 & -0.326 & -0.312 & -0.305 & $3.6 \mathrm{e}-01$ & $7.7 \mathrm{e}-02$ & $3.1 \mathrm{e}-02$ & $8.9 \mathrm{e}-03$ \\
$Z_{\mathrm{eq}}^{\mathrm{b}}$ & 2.28 & 2.17 & 2.24 & 2.26 & 2.27 & $5.0 \mathrm{e}-02$ & $1.9 \mathrm{e}-02$ & $9.8 \mathrm{e}-03$ & $3.7 \mathrm{e}-03$ \\
\hline
\end{tabular}

\section{References}

Ansorg M, Kleinwächter A and Meinel R 2003 Astron. Astrophys. 405 711-21.

Belinski V A and Zakharov V E 1978 Zh. Eksp. Teor. Fiz. 75 1953-71.

Berti E and Stergioulas N 2004 Mon. Not. R. Astron. Soc. 350 1416-30.

Ernst F J 1968a Phys. Rev. 1671175.

Ernst F J 1968b Phys. Rev. 168 1415-17.

Fodor G, Hoenselaers C and Perjés Z 1989 J. Math. Phys. 30 2252-57.

Galassi M, Davies J, Theiler J, Gough B, Jungman G, Alken P, Booth M and Rossi F 2009 GNU Scientific Library Reference Manual - Third Edition (v1.12) (Network Theory Ltd).

Harrison B K 1978 Phys. Rev. Lett. 41 1197-1200.

Hauser I and Ernst F J 1981 J. Math. Phys. 22, 1051-1063.

Kordas P 1995 Class. Quantum Grav. 12 2037-44.

Kramer D 1980 in Abstracts of contributed papers vol 1, Proc. of the 9th Int. Conf. on General Relativity and Gravitation, Jena 42-43.

Kramer D and Neugebauer G 1968 Commun. Math. Phys. 10 132-39.

Kramer D, Neugebauer G and Matos T 1991 J. Math. Phys. 32 2727-30.

Lichtenstein L 1933 Gleichgewichtsfiguren Rotierender Flüssigkeiten (Berlin: Springer).

Lindblom L 1992 Phil. Trans. R. Soc. Lond. A 340 353-64. 
Analytical approximation of the exterior gravitational field of rotating neutron stars 18

Maison D 1978 Phys. Rev. Lett. 41 521-22.

Manko V S and Ruiz E 1998 Class. Quantum Grav. 15 2007-16.

Manko V S and Sibgatullin N R 1993 Class. Quantum Grav. 10 1383-1404.

Maucher F 2008 Lösungen der Ernst Gleichung mit rationalem Achsenpotential, Master's thesis, Friedrich-Schiller-Universität, Jena.

Meinel R, Ansorg M, Kleinwächter A, Neugebauer G and Petroff D 2008 Relativistic Figures of Equilibrium (Cambridge: Cambridge University Press).

Meinel R and Neugebauer G 1995 Class. Quantum Grav. 12 2045-50.

Nelder J A and Mead R 1965 Comput. J. 7 308-13.

Neugebauer G 1979 J. Phys. A: Math. Gen. 12 L67-L70.

Neugebauer G 1980a J. Phys. A: Math. Gen. 13 L19.

Neugebauer G $1980 b$ J. Phys. A: Math. Gen. 13 1737-40.

Neugebauer G 1996 in General Relativity edited by Hall G S and Pulham J R, SUSSP Publications and Institute of Physics Publishing, Edinburgh University and The Institute of Physics, London 61-81.

Neugebauer G and Hennig J 2009 Gen. Rel. Grav. 41 2113-30.

Neugebauer G and Kramer D 1983 J. Phys. A: Math. Gen. 16 1927-36.

Neugebauer G and Meinel R 2003 J. Math. Phys. 44 3407-29.

Nozawa T, Stergioulas N, Gourgoulhon E and Eriguchi Y 1998 Astron. Astrophys. 132 431-54.

Pappas G 2009 J. Phys.: Conf. Ser. 189012028.

Sibgatullin N R 1991 Oscillations and Waves in Strong Gravitational and Electromagnetic Fields (Berlin: Springer).

Stute M and Camenzind M 2002 Mon. Not. R. Astron. Soc. 336 831-40. 